University of Nebraska - Lincoln

DigitalCommons@University of Nebraska - Lincoln

\title{
Interface proximity effects in current-perpendicular-to-plane magnetoresistance
}

\author{
R.J. Baxter \\ University of Oxford, Parks Road, Oxford OX1 3PH, United Kingdom \\ David G. Pettifor \\ University of Oxford, david.pettifor@materials.ox.ac.uk \\ Evgeny Y. Tsymbal \\ University of Nebraska-Lincoln, tsymbal@unl.edu
}

Follow this and additional works at: https://digitalcommons.unl.edu/cmrafacpub

Part of the Nanoscience and Nanotechnology Commons

Baxter, R.J.; Pettifor, David G.; and Tsymbal, Evgeny Y., "Interface proximity effects in currentperpendicular-to-plane magnetoresistance" (2007). Faculty Publications from Nebraska Center for Materials and Nanoscience. 16.

https://digitalcommons.unl.edu/cmrafacpub/16

This Article is brought to you for free and open access by the Materials and Nanoscience, Nebraska Center for (NCMN) at DigitalCommons@University of Nebraska - Lincoln. It has been accepted for inclusion in Faculty Publications from Nebraska Center for Materials and Nanoscience by an authorized administrator of DigitalCommons@University of Nebraska - Lincoln. 


\title{
Interface proximity effects in current-perpendicular-to-plane magnetoresistance
}

\author{
R. J. Baxter, ${ }^{1}$ D. G. Pettifor, ${ }^{1}$ and E. Y. Tsymbal ${ }^{2, *}$ \\ ${ }^{1}$ Department of Materials, University of Oxford, Parks Road, Oxford OX1 3PH, United Kingdom \\ ${ }^{2}$ Department of Physics and Astronomy, Center for Materials Research and Analysis, University of Nebraska, Lincoln, \\ Nebraska 68588, USA
}

(Received 2 September 2004; revised manuscript received 13 October 2004; published 20 January 2005)

\begin{abstract}
Using a tight-binding model that takes into account a realistic electronic band structure and includes defect scattering we investigate spin-dependent transport in $\mathrm{Co} / \mathrm{Cu} / \mathrm{Co}$ trilayers when current flows perpendicular to the plane. We show that resistance of the $\mathrm{Co} / \mathrm{Cu}$ interface depends on the proximity of another interface, which makes the parameters characterizing the spin-dependent interface resistance $A R_{F / N}^{*}$ and $\gamma_{F / N}$, to be dependent on the layer thickness separating the two interfaces. This leads to a decrease in the measurable quantity $S R$ $=\sqrt{\left(A R_{A P}\right)\left(A R_{A P}-A R_{P}\right)}$ with the $\mathrm{Cu}$ layer thickness and, therefore, to the departure from the series-resistor model. Here $A R_{A P}$ is the specific resistance (area $A$ times resistance $R$ ) of the trilayer when magnetizations of the two Co layers are aligned antiparallel $(A P)$ to each other, and $A R_{P}$ is the specific resistance when the layer magnetizations are aligned parallel $(P)$. We demonstrate that recent experimental data on currentperpendicular-to-plane transport in $\mathrm{Co} / \mathrm{Cu} / \mathrm{Co}$ spin valves can be explained by the interface proximity effects without introducing a finite spin-diffusion length.
\end{abstract}

DOI: 10.1103/PhysRevB.71.024415

\section{INTRODUCTION}

The phenomenon of giant magnetoresistance (GMR) has been observed in magnetic metallic multilayers in two geometries: current in the plane of the layers ${ }^{1}$ (CIP) and current perpendicular to the plane (CPP). ${ }^{2}$ Although, due to the small multilayer film thickness, experiments within the CPP geometry are much more delicate, they can provide important information about the mechanisms of spin-dependent scattering (for a recent review on GMR see Ref. 3).

The majority of experiments on CPP GMR are interpreted in terms of the series-resistor model, in which there are no relevant lengths except the layer thicknesses. The seriesresistor model is justified for free electrons when the spindiffusion length is large compared to the layer thicknesses. ${ }^{4}$ The series-resistor model can be qualitatively understood by the following arguments. When the elastic mean-free path $\lambda$ is short compared to the layer thicknesses, each layer can be considered as a separate resistor for the current flowing perpendicular to the plane of the multilayer. At the other extreme, when $\lambda$ is very long, the probability of scattering is the sum of the scattering probabilities within each layer. Therefore, the conductivity becomes self-averaging, which leads to resistors in series, as in the first case, making the mean-free path irrelevant to CPP GMR.

These arguments become, however, invalid when a realistic band structure of the metal layers comprising a GMR multilayer is taken into account. The presence of potential steps at the interfaces leads to interface proximity effects that break the series-resistor model when the layer thickness is less or comparable to the mean-free path. The dependence of the interface resistance on the proximity of other interfaces was found theoretically within a simple tight-binding model, ${ }^{5}$ for realistic tight-binding bands in a $\mathrm{Co} / \mathrm{Cu}$ multilayer, ${ }^{6}$ and also using first-principle calculations for disordered $\mathrm{Co} / \mathrm{Cu}$ interfaces. ${ }^{7}$ It was demonstrated analytically that the interface resistance is affected by the exponential terms in the electrochemical potential that decays at a rate comparable to the mean-free path. ${ }^{8,9}$ Experimentally, it was
PACS number(s): 75.70.Cn, 75.47.De, 73.50.-h, 73.40.-c

found that the magnitude of CPP GMR in $\mathrm{Co} / \mathrm{Cu}$ multilayers is sensitive to the ordering of the magnetic layers evidencing the presence of the "mean-free-path effects." 10

In CPP GMR the quantity measured is either the conductance per unit area or its inverse, the specific resistance $A R$, where $A$ is the area through which the current flows. The magnitude of GMR is defined by the ratio $\left(A R_{A P}\right.$ $\left.-A R_{P}\right) / A R_{A P}=\triangle A R / A R_{A P}$, where indices "P" and "AP" refer to the parallel and antiparallel magnetization of the multilayer, respectively. The series-resistor model, elaborated by Lee et al. ${ }^{11}$ to include spin-dependent bulk resistivities and interface resistances, expresses these quantities for a multilayer with $N$ bilayers as follows:

$$
\begin{gathered}
A R_{\mathrm{AP}}=N\left(\rho_{N} t_{N}+\rho_{F}^{*} t_{F}+2 A R_{F / N}^{*}\right), \\
A \Delta R=N^{2}\left(\beta_{F} \rho_{F}^{*} t_{F}+2 \gamma_{F / N} A R_{F / N}^{*}\right)^{2} / A R_{\mathrm{AP}} .
\end{gathered}
$$

Here $\rho_{N}$ is the resistivity of the nonmagnetic layer, $\rho_{F}^{*}$ $=\rho_{F} /\left(1-\beta_{F}^{2}\right)$ is the "enhanced" resistivity of the ferromagnetic layer, $A R_{F / N}^{*}=A R_{F / N} /\left(1-\gamma_{F / N}^{2}\right)$ is the "enhanced" interface resistance, $\beta_{F}$ is the scattering spin-asymmetry parameter for the bulk, and $\gamma_{F / N}$ for the interface. $\beta_{F}$ is related to the spin asymmetry of the bulk scattering within the ferromagnetic layer $\alpha_{F}=\rho_{F}^{\downarrow} / \rho_{F}^{\uparrow}$ by the expression $\alpha_{F}=\left(1+\beta_{F}\right) /\left(1-\beta_{F}\right) \cdot \gamma_{F / N}$ is related to the spin asymmetry of the interface scattering at the FM/NM interfaces $\alpha_{F / N}$ $=A R_{F / N}^{\downarrow} / A R_{F / N}^{\uparrow}$ by the expression $\alpha_{F / N}=\left(1+\gamma_{F / N}\right) /\left(1-\gamma_{F / N}\right)$.

When the series-resistor model is used to interpret experiment data, the interfaces are assumed to be described using only two parameters, namely, $A R_{F / N}^{*}$ and $\gamma_{F / N}$, which are independent of the layer thickness separating the interfaces. This makes the quantity

$$
S R=\sqrt{\left(A R_{\mathrm{AP}}\right)(A \Delta R)}=N\left(\beta_{F} \rho_{F}^{*} t_{F}+2 \gamma_{F / N} A R_{F / N}^{*}\right)
$$

independent of the parameters characterizing the spacer layer $\rho_{N}$ or $t_{N}$. The two terms within the $S R$ on the left-hand side of Eq. (3) can be measured. Thus, for the series-resistor model, 
a plot of $S R$ versus $t_{N}$ for a series of samples with fixed $t_{F}$ and fixed $N$, but varying $t_{N}$, should yield a horizontal line. If interface proximity effects are present, $S R$ should deviate from the horizontal line.

The search for deviations from such a horizontal line was recently performed by Chiang et al. ${ }^{12}$ for $\mathrm{Co} / \mathrm{Cu} / \mathrm{Co}$, and $\mathrm{Co} / \mathrm{Ag} / \mathrm{Co}$, and $\mathrm{Co} / \mathrm{Au} / \mathrm{Co}$ exchange-biased spin-valves. In all cases sizable deviations were found. However, these deviations were largely interpreted using the Valet-Fert model, ${ }^{13}$ which takes into account spin-flipping of free electrons but ignores a realistic band structure of the multilayer and, therefore, interface proximity effects. Only for $\mathrm{Co} / \mathrm{Cu} / \mathrm{Co}$ spin valves the decrease, which was larger than expected within the Valet-Fert model was found; for $\mathrm{Co} / \mathrm{Ag} / \mathrm{Co}$ and $\mathrm{Co} / \mathrm{Au} / \mathrm{Co}$ spin-valves, the decreases were entirely explained using finite spin-diffusion lengths. The authors concluded that due to uncertainties in these lengths and the data, interface proximity effects (mean-free-path effects) cannot be ruled out.

In this paper we use a model that takes into account a realistic band structure of the $\mathrm{Co} / \mathrm{Cu} / \mathrm{Co}$ spin valves and includes defect scattering. ${ }^{14}$ This model was used successfully to interpret experiment data on thickness-dependent $\mathrm{CIP}$ conductivity in $\mathrm{Co} / \mathrm{Cu} / \mathrm{Co}$ spin-valves measured in situ ${ }^{15}$ to explain experimentally observed signs of the thermoelectric power in $\mathrm{Co} / \mathrm{Cu}$ and $\mathrm{Fe} / \mathrm{Cr}$ multilayers, ${ }^{16}$ and to elucidate the strong dependence of the CPP GMR on the order of magnetic layers in a $\mathrm{Co} / \mathrm{Cu}$ multilayer. ${ }^{10}$ We show that resistance of the $\mathrm{Co} / \mathrm{Cu}$ interface depends on the proximity of another interface that causes the parameters characterizing the spin-dependent interface resistance, $A R_{F / N}^{*}$ and $\gamma_{F / N}$, to depend on the layer thickness separating the two interfaces. This leads to a decrease in SR with the $\mathrm{Cu}$ layer thickness and, therefore, to the departure from the seriesresistor model. We demonstrate that the experiment data of Chiang et al. ${ }^{12}$ for $\mathrm{Co} / \mathrm{Cu} / \mathrm{Co}$ spin valves can be explained by the interface proximity effect without introducing a finite spin diffusion length.

\section{METHOD OF CALCULATION}

We describe the electronic structure of a $\mathrm{Co} / \mathrm{Cu} / \mathrm{Co}$ trilayer using a realistic multiband tight-binding model, which accounts for $s, p$, and $d$ orbitals with their full hybridization and spin polarization. ${ }^{14}$ Disorder is introduced in this model as a random variation in the on-site atomic energies of the $\mathrm{Cu}$ and $\mathrm{Co}$ atoms, with a uniform distribution of standard deviation $\delta .{ }^{17}$ For calculating the conductance the disordered $\mathrm{Co} / \mathrm{Cu} / \mathrm{Co}$ trilayer is placed between two perfect semiinfinite Co leads [Fig. 1(a)], where stacking is in the [001] direction and the $\mathrm{Co}$ and $\mathrm{Cu}$ layers are assumed to have the fcc structure with a lattice parameter $a$ equal to that of bulk $\mathrm{Cu}, a=0.361 \mathrm{~nm}$. For each disorder configuration, the conductance is calculated using the Kubo formula within the real-space technique. ${ }^{6}$ First, the matrix elements of the surface Green's function for the bulk Co (001) are calculated, and then disordered layers are added to the left lead in order to grow the trilayer. The Dyson equation is solved numerically to find the Green's function for each of the added layers

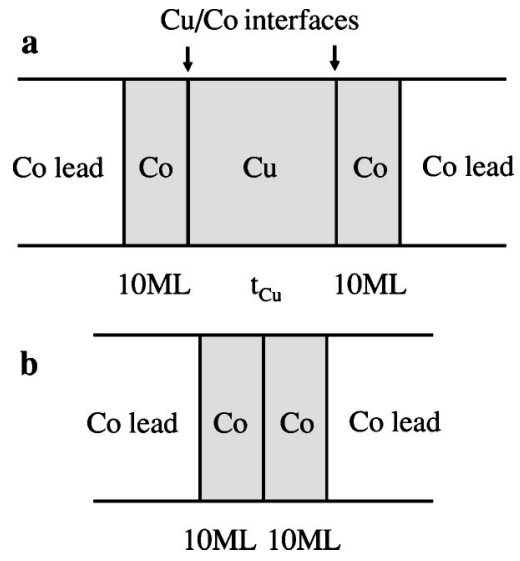

FIG. 1. Geometry used in calculations of the resistances of the $\mathrm{Co} / \mathrm{Cu} / \mathrm{Co}$ trilayer and $\mathrm{Co} / \mathrm{Cu}$ interfaces. Gray contrast indicates disorder.

in turn. Finally, when the last layer is added, it is bonded to the right lead to obtain the Green's function of the full system, which is used to calculate the conductance. Periodic boundary conditions are imposed on a cell of $4 a \times 4 a$ in the transverse direction, $4 k$ points are calculated in the Brillouin zone, and the conductance is averaged over 12 random configurations of disorder.

\section{RESULTS AND DISCUSSION}

First, we calculate the resistance of the $\mathrm{Co} / \mathrm{Cu}\left(t_{\mathrm{Cu}}\right) / \mathrm{Co}$ trilayer as a function of the thickness of the $\mathrm{Cu}$ layer $t_{\mathrm{Cu}}$. The geometry of the system is shown schematically in Fig. 1(a). The thickness of the disordered Co layers is assumed to be 10 monolayers (ML), which is equivalent to $1.8 \mathrm{~nm}$. In this calculation the disorder parameter $\delta=0.35 \mathrm{eV}$ is chosen to be the same in the bulk of the $\mathrm{Co}$ and $\mathrm{Cu}$ layers and at the interfaces. This value of $\delta$ provides bulk resistivities $\rho_{\text {Co }}$ $\approx 60 \mathrm{n} \Omega \mathrm{m}$ and $\rho_{\mathrm{Cu}} \approx 15 \mathrm{n} \Omega \mathrm{m}$ similar to those observed experimentally. ${ }^{12}$ Figure 2 (a) shows results of the calculation for parallel (P) and antiparallel (AP) magnetization of the trilayer. Nonlinearity of the spin-resolved resistances is evident from the figure, which is especially pronounced for the AP configuration. This deviation from the Ohmic behavior is due to the proximity of the two $\mathrm{Co} / \mathrm{Cu}$ interfaces. In order to elucidate the calculated data we fit the spin-resolved resistances using the following expression:

$$
A R=A R_{0}+2 \rho_{\mathrm{Cu}} t_{\mathrm{Cu}}+A R_{1} \exp \left(-\frac{t_{\mathrm{Cu}}}{\lambda}\right) .
$$

Here the first term $A R_{0}$ includes the interface resistances and the ballistic conductance of the leads, the second term reflects the bulk resistance of the $\mathrm{Cu}$ layer, and the third term describes nonlinearity caused by interface proximity. The parameter $\lambda$ is the effective mean-free path, which depends not only on the properties of the spacer layer (such as the electronic structure and type and density of scatterers) but also on the electronic structure of the interfaces adjacent to this layer.

Table I contains the values of the constants in Eq. (4) for each of the three curves fitted. The linear part of Eq. (4) 


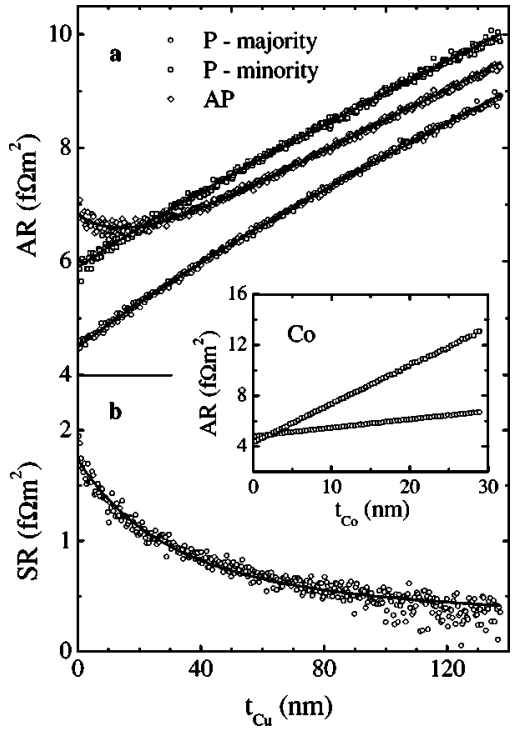

FIG. 2. Resistance $A R$ of $\mathrm{Co}(10 \mathrm{ML}) / \mathrm{Cu}\left(t_{\mathrm{Cu}}\right) / \mathrm{Co}(10 \mathrm{ML})$ trilayer (a) and $S R \sqrt{\left(A R_{A P}\right)\left(A R_{A P}-A R_{P}\right)}$, (b) as a function of $\mathrm{Cu}$ layer thickness, $t_{\mathrm{Cu}}$, in the presence of bulk disorder $\delta=0.35 \mathrm{eV}$. Solid lines display fit to the calculated data using Eq. (4). The inset shows the majority- (circles) and minority- (squares) spin resistance for a $\operatorname{Co}(10 \mathrm{ML}) / \mathrm{Co}\left(t_{\mathrm{Co}}\right) / \mathrm{Co}(10 \mathrm{ML})$ trilayer demonstrating a linear variation of $A R$ in the absence of interfaces.

represents the Ohmic behavior and has the same gradient for all three. The larger value of $A R_{0}$ for minority spins reflects the larger $\mathrm{Co} / \mathrm{Cu}$ interface resistance, which is not compensated by the greater ballistic conductance for this spin orientation. ${ }^{18}$ The exponential component of Eq. (4) reflects a deviation from linearity related to the variation in the interface resistance because of the proximity of the two $\mathrm{Cu} / \mathrm{Co}$ interfaces. The fact that the constant $A R_{1}$ is negative for the $\mathrm{P}$ magnetization and positive for the AP magnetization shows that this effect differs for the two configurations, as will be discussed below.

The value of $\lambda$ determines the rate of convergence of the exponential part of Eq. (4) and, hence, the return to linearity and the Ohmic regime of conduction. This occurs fastest for minority-spin electrons and slowest for the AP configuration, with majority-spin electrons in the $\mathrm{P}$ configuration lying in between. The dependence of $\lambda$ on spin and magnetization configuration originates from a different distribution of electrons entering the $\mathrm{Cu}$ spacer layer from the Co lead over transverse wave vectors $\mathbf{k}_{\|}$within the interface Brillouin zone $^{19}$ and from the state dependence of the mean-free path. Majority-spin electrons have a longer mean-free path due to the higher probability of transmission for electrons with

TABLE I. Fitting parameters for calculated data presented in Fig. 2(a) and 4.

\begin{tabular}{ccccc}
\hline \hline & $A R_{0}\left(\mathrm{f} \Omega \mathrm{m}^{2}\right)$ & $A R_{1}\left(\mathrm{f} \Omega \mathrm{m}^{2}\right)$ & $A R_{i}\left(\mathrm{f} \Omega \mathrm{m}^{2}\right)$ & $\lambda(\mathrm{nm})$ \\
\hline P majority & 5.21 & -0.70 & 0.39 & 53 \\
P minority & 6.25 & -0.31 & 1.18 & 39 \\
AP & 5.73 & 1.11 & 0.79 & 17 \\
\hline \hline
\end{tabular}

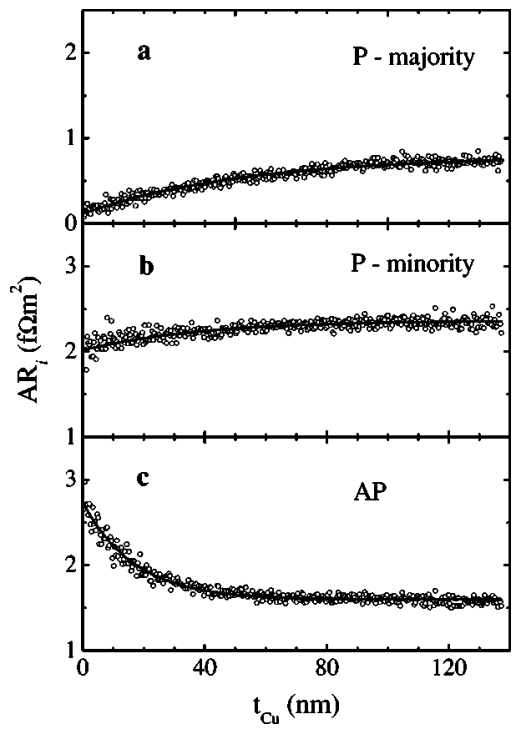

FIG. 3. Spin-resolved resistance of two $\mathrm{Co} / \mathrm{Cu} / \mathrm{Co}$ interfaces separated by disordered $\mathrm{Cu}$ layer of thickness $t_{\mathrm{Cu}}$ for (a) majorityand (b) minority- spin electrons for (c) $P$ magnetization and $A P$ magnetization. Solid lines display fit to the calculated data using Eq. (5).

smaller $\mathbf{k}_{\|}$, which have higher velocity. Using the Drude formula for conductivity, $\sigma=\left(e^{2} / h\right)\left(k_{F}^{2} / 3 \pi\right) \bar{\lambda}$, and the known resistivity value for bulk $\mathrm{Cu}, \rho_{\mathrm{Cu}} \approx 15 \mathrm{n} \Omega \mathrm{m}$, we estimate the average mean-free path in $\mathrm{Cu}$ to be $\bar{\lambda} \approx 44 \mathrm{~nm}$. This value lies somewhat in between the values of $\lambda$ found for majorityand minority-spin electrons, reflecting the averaging over states with different $\mathbf{k}_{\|}$.

Figure 2(b) shows the SR quantity (3) as a function of the $\mathrm{Cu}$ layer thickness. According to the series-resistor model, this quantity should be independent of $t_{\mathrm{Cu}}$. However, in contrast, our results show a large initial decrease in the SR with increasing $t_{\mathrm{Cu}}$, which is related to the nonlinear behavior of the $\mathrm{P}$ and AP resistances shown in Fig. 2(a). The breakdown in the series-resistor model stems from the fact that the $\mathrm{Cu} / \mathrm{Co}$ interface resistance is assumed to be constant, regardless of the $\mathrm{Cu}$ thickness.

In order to demonstrate explicitly the dependence of the $\mathrm{Co} / \mathrm{Cu}$ interface resistance on the proximity of the other interface, we performed additional calculations. Figures 1(a) and 1(b) summarize the geometry of these calculations. The total resistance of the two $\mathrm{Cu} / \mathrm{Co}$ interfaces, labeled in Fig. 1(a), is obtained in two steps. First, the resistance of Fig. 1(b) is subtracted from 1(a), leaving the resistance of the two $\mathrm{Cu} / \mathrm{Co}$ interfaces plus the resistance of the $\mathrm{Cu}$ layer, obtained as a function of $t_{\mathrm{Cu}}$. Second, the resistance of the $\mathrm{Cu}$ layer, $A R_{\mathrm{Cu}}=\rho_{\mathrm{Cu}} t_{\mathrm{Cu}}$, is subtracted from this value, leaving the total resistance of the two interfaces.

Figure 3 shows the total resistance of both $\mathrm{Cu} / \mathrm{Co}$ interfaces against the $\mathrm{Cu}$ layer thickness, for $\mathrm{P}$ and $\mathrm{AP}$ magnetizations. It is clear from the figure that this resistance is not independent of $t_{\mathrm{Cu}}$. There are strong variations, particularly at lower $t_{\mathrm{Cu}}$, when the interfaces are in close proximity. The layer-thickness-dependent interface resistance is the origin of the breakdown in the series-resistor model. As the $\mathrm{Cu}$ layer 
thickness becomes sufficiently large the resistance of the interfaces tends toward a constant value, so the series-resistor model behavior is recovered.

The most striking variation in the interface resistance is in the AP case, as can be seen in Fig. 3(c). The interface resistance shows a strong initial decrease with $t_{\mathrm{Cu}}$. This is because of the very distinct electronic structures of the majority and minority bands in $\mathrm{Co}$. Scattering by disorder in the $\mathrm{Cu}$ layer assists the electrons that have passed the first $\mathrm{Co} / \mathrm{Cu}$ interface to be transmitted across the second $\mathrm{Cu} / \mathrm{Co}$ interface, and hence the interface resistance decreases with increasing $t_{\mathrm{Cu}}$. The situation is, however, different for the parallel case. As is seen in Figs. 3(a) and 3(b), the interface resistance increases with the $\mathrm{Cu}$ layer thickness for both majority and minority electrons in the $\mathrm{P}$ configuration, in contrast to the decrease in the AP case. This is because quantum-well bound states are created in the $\mathrm{Cu}$ layer when it is placed between the Co leads of higher electronic potential. These bound states do not contribute to the conductance at small $\mathrm{Cu}$ thickness. Therefore, majority-spin electrons, due to similarity of the band structures of $\mathrm{Cu}$ and $\mathrm{Co}$, traverse the thin $\mathrm{Cu}$ layer almost with no scattering, making the interface resistance very small [see Fig. 3(a)]. However, as $t_{\mathrm{Cu}}$ increases, the defect scattering redistributes the current-carrying electrons between the conducting and bound states, and the interface resistance increases. Minority-spin electrons display a less pronounced departure from linearity than majority-spin electrons. This is because of disorder, which intermixes the closely lying minority Co $d$ bands and smears out the potential well. ${ }^{6}$

The calculated data shown in Fig. 3 can be fitted using the expression

$$
A R=2 A R_{i}+A R_{1} \exp \left(-\frac{t_{\mathrm{Cu}}}{\lambda}\right)
$$

in which $A R_{1}$ and $\lambda$ have the same values as in Eq. (4). The fit allows us to find the values of the interface resistance $A R_{i}$ in the asymptotic limit of a thick spacer layer. The results are presented in Table I. It is not surprising that within a statistical error the value of $A R_{i}$ for the AP configuration is equal to the average of the $A R_{i}$ values for the majority and minority electrons in the $\mathrm{P}$ configuration, reflecting the fact that for sufficiently large $\mathrm{Cu}$ layer thickness, the series-resistor model behavior is recovered.

The asymptotic value of the interface resistance obtained in our calculation for majority-spin electrons, $0.39 \mathrm{f} \Omega \mathrm{m}^{2}$, is in very good agreement with the value of $0.35 \mathrm{f} \Omega \mathrm{m}^{2}$ reported in Ref. 18 for a single $\mathrm{Co} / \mathrm{Cu}(001)$ interface. On the other hand, the asymptotic value of the interface resistance for majority-spin electrons in our calculation, $1.18 \mathrm{f} \Omega \mathrm{m}^{2}$, is much less than the value of $1.9 \mathrm{f} \Omega \mathrm{m}^{2}$ in Ref. 18. The reason for such a strongly reduced interface resistance is the presence of disorder in our model. A significant mismatch in the band structures of the minority-spin electrons in $\mathrm{Co}$ and $\mathrm{Cu}$ leads to a low transmission coefficient through the $\mathrm{Co} / \mathrm{Cu}$ interface for the minority spins in the absence of disorder. ${ }^{19}$ This is the condition at which the diffuse scattering assists conduction, thereby reducing the resistance. ${ }^{20}$

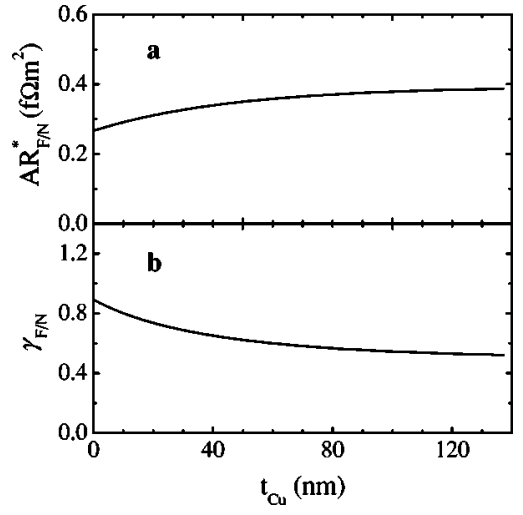

FIG. 4. Parameters of the spin-dependent $\mathrm{Co} / \mathrm{Cu}$ interface resistance as a function of disordered $\mathrm{Cu}$ layer thickness: (a) enhanced interface resistance $A R_{F / N}^{*}$ and (b) spin-asymmetry scattering parameter $\gamma_{F / N}$.

Variation of the spin-dependent interface resistances with the thickness of the layer separating the two interfaces causes the parameters characterizing the interface resistance, $A R_{F / N}^{*}$ and $\gamma_{F / N}$, to depend on the proximity of the interfaces. Figure 4 shows the dependence of $A R_{F / N}^{*}$ and $\gamma_{F / N}$ on $t_{\mathrm{Cu}}$ recalculated from the fitting curves in Figs. 3(a) and 3(b). Not unexpectedly, these parameters vary quite considerably with the $\mathrm{Cu}$ layer thickness. $A R_{F / N}^{*}$ increases from 0.27 to $0.39 \mathrm{f} \Omega \mathrm{m}^{2} . \gamma_{F / N}$ drops from 0.88 to 0.52 , reflecting a decrease in the spin asymmetry with increasing $t_{\mathrm{Cu}}$. These calculated values of $A R_{F / N}^{*}$ and $\gamma_{F / N}$ are consistent with the experiment values. ${ }^{21}$ The layer thickness dependence of these parameters might partly explain the spread in the values of these parameters obtained by different experimental groups, although other factors may also play a role (see, e.g., Ref. 21 and references therein).

The interface proximity effect is sensitive to the degree of disorder at the interfaces because scattering by this disorder averages the transmission probability over different transverse wave vectors $\mathbf{k}_{\|}$, improving the conditions for applicability of the series-resistor model. Due to interface roughness and interdiffusion it is likely that the interface disorder is stronger than the bulk disorder. The influence of the interface disorder is explored in Fig. 5, where three SR curves are plotted as a function of the $\mathrm{Cu}$ thickness. In the calculation of each of the curves, the bulk disorder parameter was kept

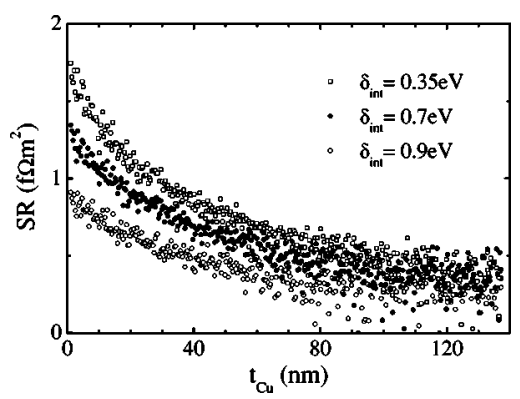

FIG. 5. Calculated $S R=\sqrt{\left(A R_{A P}\right)\left(A R_{A P}-A R_{P}\right)}$ vs $\mathrm{Cu}$ layer thickness for different values of disorder parameter $\delta_{i n t}$ at $\mathrm{Co} / \mathrm{Cu}$ interfaces. 


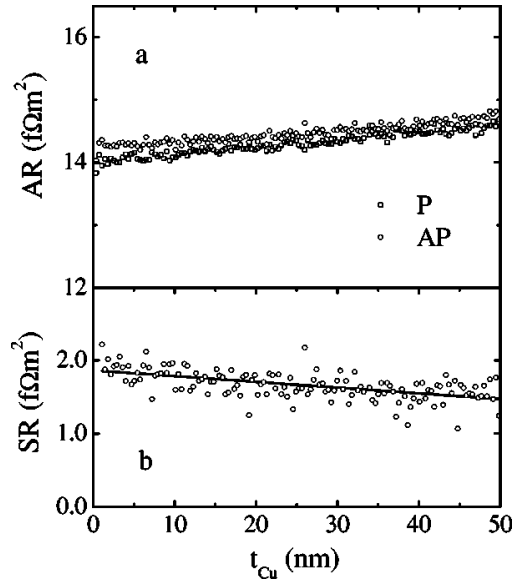

FIG. 6. Resistance for parallel (P) and antiparallel (AP) configuration of (a) a $\mathrm{Co} / \mathrm{Cu} / \mathrm{Co}$ spin valve and $S R$ vs (b) Co layer thickness for $\delta=0.35 \mathrm{eV}$ and $\delta_{\text {int }}=1.05 \mathrm{eV}$ assuming that an additional resistor in series is included to fit the resistance measured in Ref. 12. The solid line has the slope of $-0.007 \mathrm{f} \Omega \mathrm{m}^{2} / \mathrm{nm}$, which is the best fit to the experimental data (Ref. 12).

to be constant $\delta=0.35 \mathrm{eV}$, whereas the disorder within one $\mathrm{Cu}$ monolayer and one $\mathrm{Co}$ monolayer on each side of a $\mathrm{Cu} / \mathrm{Co}$ interface $\delta_{\text {int }}$ was varied.

The three SR curves in Fig. 5 exhibit qualitatively similar behavior, showing a decrease in SR with increasing $t_{\mathrm{Cu}}$. It is seen that increasing interface disorder leads to a flattening of the SR curve, resulting in better agreement with the seriesresistor model, which requires SR to be constant. Nevertheless, even for large values of interface disorder we see a sizable variation in SR.

The results of our calculations can be compared with the experimental data obtained for $\mathrm{Co} / \mathrm{Cu} / \mathrm{Co}$ spin valves by Chiang et al. ${ }^{12}$ These data show a gradual decrease in SR with $t_{\mathrm{Cu}}$ and are fitted by a straight line with a slope of $-0.007 \mathrm{f} \Omega \mathrm{m}^{2} / \mathrm{nm}$ and a maximum value of the $\mathrm{SR}$ at zero thickness of about $2 \mathrm{f} \Omega \mathrm{m}^{2}$ (see Fig. 6 in Ref. 12). The SR magnitude depends on the total resistance of the system, which includes other resistors (such as a pinning layer) not considered in our calculation. Therefore, in order to correlate our results with the experimental data we first added a resistor in series to our data to obtain the total resistance in the range of $14 \mathrm{f} \Omega \mathrm{m}^{2}$ close to the experimental values. Then, we varied the interface disorder parameter $\delta_{i n t}$ keeping other parameters fixed to obtain the experimentally measured slope of the SR quantity versus $t_{\mathrm{Cu}}$ of $-0.007 \mathrm{f} \Omega \mathrm{m}^{2} / \mathrm{nm}$. The best agreement was found for $\delta_{\text {int }}=1.05 \mathrm{eV}$. The results are shown in Fig. 6 in the same range of data points as in Ref. 12. As is seen from Fig. 6(a), such a strong interface disorder in experimental samples $\left(\delta_{i n t}=1.05 \mathrm{eV}\right)$ makes deviations from the series resistor model less pronounced than those in Fig. 2(a), in which $\delta_{\text {int }}=\delta=0.35 \mathrm{eV}$. This is due to the strong diffuse interface scattering that destroys the electron coherence, making the interference of scattered electrons from different interfaces less evident. In order to make sense of the magnitude of the parameter $\delta_{\text {int }}$, we note that changing $\delta$ from 0.35 to $1.05 \mathrm{eV}$ enhances bulk resistivity of $\mathrm{Cu}$ by an order of magnitude. Nevertheless, the deviations from the series resistor cannot be ignored and lead to a nonnegligible decrease is SR, as is seen from Fig. 6(b). The solid line in this figure has a slope of $-0.007 \mathrm{f} \Omega \mathrm{m}^{2} / \mathrm{nm}$ showing the agreement with experiment. We note that the absolute values of the SR are also consistent with experimental data, demonstrating the correct magnitude of $\left(A R_{A P}-A R_{P}\right)$ and, consequently, the GMR ratio in our calculation. We conclude, therefore, that the experimental data presented in paper [12] on CPP GMR in $\mathrm{Co} / \mathrm{Cu} / \mathrm{Co}$ spin valves can be explained by the interface proximity effect without introducing a finite spin diffusion length.

\section{CONCLUSIONS}

Using a tight-binding model, which takes into account realistic electronic band structure and includes defect scattering, we have investigated spin-dependent transport in $\mathrm{Co} / \mathrm{Cu} / \mathrm{Co}$ trilayers when current flows perpendicular to the plane. Our results show that $S R=\sqrt{\left(A R_{A P}\right)\left(A R_{A P}-A R_{P}\right)}$ varies with the thickness of the $\mathrm{Cu}$ layer, which is in contradiction to the series-resistor model, where this quantity is assumed to be constant. The variation in SR is most striking at smaller thickness of the nonmagnetic layer and is related to the proximity of the $\mathrm{Cu} / \mathrm{Co}$ interfaces. When the $\mathrm{Cu}$ spacer layer thickness is smaller than the mean-free path, the total resistance of the two interfaces is considerably different than at large $\mathrm{Cu}$ thicknesses. This variation in the resistance of the $\mathrm{Co} / \mathrm{Cu}$ interface with the $\mathrm{Cu}$ layer thickness causes the parameters characterizing the spin-dependent interface resistance, $A R_{F / N}^{*}$ and $\gamma_{F / N}$, to depend on the layer thickness separating the two interfaces, thereby demonstrating departure from the series-resistor model. Interface proximity effects are also seen when enhanced interface disorder is introduced into the calculations, although it becomes less pronounced with increasing disorder.

The results of our calculations are consistent with the experimental data of Chiang et al. ${ }^{12}$ on CPP GMR in $\mathrm{Co} / \mathrm{Cu} / \mathrm{Co}$ spin valves. We show that these data can be explained by interface proximity effects without introducing a finite spin-diffusion length. This fact suggests that the estimates for the spin-diffusion length based on a model that ignores the interface proximity effects in CPP GMR may lead to underestimated values of the spin-diffusion length. It would be highly desirable to develop an experimental method for measuring the spin-diffusion length with no involvement of any model.

\section{ACKNOWLEDGMENTS}

The authors thank Jack Bass for many helpful discussions. This work was funded by the EPSRC. We are also pleased to acknowledge support from NSF (Grant No. DMR0203359) and from NSF-MRSEC (Grant No. DMR0213808), the Nebraska Research Initiative, and W. M. Keck Foundation. Computations were performed in the Materials Modelling Laboratory, University of Oxford, and in the Department of Physics, University of Nebraska. R.J.B. thanks the Department of Physics, University of Nebraska, for the kind hospitality during his stay. 
*Electronic address: tsymbal@unl.edu

${ }^{1}$ M. N. Baibich, J. M. Broto, A. Fert, F. Nguyen Van Dau, F. Petroff, P. Eitenne, G. Greuzet, A. Friederich, and J. Chazelas, Phys. Rev. Lett. 61, 2472 (1988).

${ }^{2}$ W. P. Pratt, Jr., S.-F. Lee, J. M. Slaughter, R. Loloee, P. A. Schroeder, and J. Bass, Phys. Rev. Lett. 66, 3060 (1991).

${ }^{3}$ E. Y. Tsymbal and D. G. Pettifor, in Solid State Physics (Academic Press, San Diego, 2001), Vol. 56, pp. 113-237.

${ }^{4}$ S. Zhang and P. M. Levy, J. Appl. Phys. 69, 4786 (1991); H. E. Camblong, S. Zhang, and P. M. Levy, Phys. Rev. B 47, 4735 (1993).

${ }^{5}$ E. Y. Tsymbal and D. G. Pettifor, Phys. Rev. B 61, 506 (2000).

${ }^{6}$ E. Y. Tsymbal, Phys. Rev. B 62, R3608 (2000).

${ }^{7}$ K. Xia, P. J. Kelly, G. E. W. Bauer, I. Turek, J. Kudrnovsky, and V. Drchal, Phys. Rev. B 63, 064407 (2001).

${ }^{8}$ W. H. Butler, X. G. Zhang, and J. M. MacLaren J. Appl. Phys. 87, 5173 (2000).

${ }^{9}$ A. Shpiro and P. M. Levy, Phys. Rev. B 63, 014419 (2001).

${ }^{10}$ D. Bozec, M. A. Howson, B. J. Hickey, S. Shatz, N. Wiser, E. Y. Tsymbal, and D. G. Pettifor, Phys. Rev. Lett. 85, 1314 (2000).

${ }^{11}$ S.-F. Lee, W. P. Pratt, R. Loloee, P. A. Schroeder, and J. Bass,
Phys. Rev. B 46, 548 (1992); S.-F. Lee, W. P. Pratt, Q. Yang, P. Holody, R. Loloee, P. A. Schroeder, and J. Bass, J. Magn. Magn. Mater. 118, L1 (1993).

${ }^{12}$ W. C. Chiang, C. Ritz, K. Eid, R. Loloee, W. P. Pratt, and J. Bass, Phys. Rev. B 69, 184405 (2004).

${ }^{13}$ T. Valet and A. Fert, Phys. Rev. B 48, 7099 (1993).

${ }^{14}$ E. Y. Tsymbal and D. G. Pettifor, Phys. Rev. B 54, 15314 (1996).

${ }^{15}$ W. E. Bailey, S. W. Wang, and E. Y. Tsymbal, Phys. Rev. B 61, 1330 (2000).

${ }^{16}$ E. Y. Tsymbal, D. G. Pettifor, J. Shi, and M. B. Salamon, Phys. Rev. B 59, 8371 (1999).

${ }^{17} \mathrm{We}$ note that in our previous papers the disorder parameter was denoted by $\gamma$. Here we change the notation to avoid confusion with the parameter $\gamma_{F / N}$ describing spin asymmetry of the interface scattering.

${ }^{18}$ K. M. Schep, J. B. A. N. van Hoof, P. J. Kelly, G. E. W. Bauer, and J. E. Inglesfield, Phys. Rev. B 56, 10805 (1997).

${ }^{19}$ M. Stiles, J. Appl. Phys. 79, 5805 (1996).

${ }^{20}$ S. Zhang and P. M. Levy, Phys. Rev. B 57, 5336 (1998).

${ }^{21}$ J. Bass and W. P. Pratt Jr., J. Magn. Magn. Mater. 200, 274 (1999). 